\title{
Test equipment and its effect on the calibration of instrument transformers
}

\author{
Enrico Mohns and Peter Räther \\ Physikalisch-Technische Bundesanstalt (PTB), Bundesallee 100, 38116 Braunschweig, Germany \\ Correspondence: Enrico Mohns (enrico.mohns@ptb.de)
}

Received: 5 December 2017 - Revised: 5 April 2018 - Accepted: 10 April 2018 - Published: 7 May 2018

\begin{abstract}
State-approved test centres in Germany supplying accuracy tests for instrument transformers must, in the future, provide measurement uncertainty budgets for their quality management systems. In this work, the cause of ratio error and phase displacement of instrument transformers are therefore discussed. The traceability to the national standards of PTB, the attainable uncertainty, and the permitted error limits of test equipment for testing instrument transformers are presented. Finally, an example of an uncertainty budget for a current transformer of the class $0,2 \mathrm{~S}$ is given.
\end{abstract}

\section{Introduction}

Instrument transformers that are used for the billing of electric energy are connected to the energy meters if the currents or the voltages to be measured are too high for the meters. This is generally the case when the energy transmitted has to be measured in the medium-voltage or the high-voltage power grids, since meters which can be directly connected are designed for the nominal voltages of the low-voltage grid. However, in applications in which the maximum expected currents are higher than $100 \mathrm{~A}$, transformer-connected meters are used with current transformers connected before them. The task of the instrument transformers is thus to reproduce the high primary voltage (or current) to be measured as a low secondary voltage (or current) which is easy for the meters to detect. Ideally, the reproduced secondary quantities should be exactly proportional and in phase with the primary quantities. In practice, however, deviations always occur. These deviations, which are caused by the instrument transformers, lead to deviations of the electric energy measured by the transformer-connected meter. In the different standardized classes, the accuracy achieved under all conditions of use is reflected as the measure for the metrological quality of an instrument transformer.

In the past, state-approved test centres therefore proved compliance with the respective accuracy classes of the transformers by means of verification before the transformers could be installed in the grids. Before this can happen, the instrument transformer has to obtain type approval. The test equipment used at the test centres are calibrated at PTB at regular intervals and verified with regard to their compliance with minimum requirements on their accuracy. This procedure is called a test ("Prüfung") as the result of this test is a test certificate which contains both the results of the calibration and a statement that the test equipment is verified according to the requirements, which are laid down in the PTB Testing Instructions (PTB, 1979). In the state-approved test centres, this procedure, based on compliance with limit values and on the thus proven traceability of the test equipment to the national standards, ensured - even without exhaustive mathematical evidence of the accuracy achieved by the transformer measuring system set up with the test equipment - that the deviations of the instrument transformers to be verified are in sufficient agreement with the International System of Units (Sommer et al., 2001).

With the new Measures and Verification Act (MEG, 2015), however, the metrological prerequisites are also changing. In the test centres certifying the conformity of an instrument transformer, not only must the test equipment be traced to national standards via a test. Within the scope of the quality management system, the test centre must also yield mathematical evidence of the uncertainty achieved during the process of an instrument transformer conformity assessment. This piece of evidence is given in the form of an uncertainty budget pursuant to the rules (GUM, 2008). Based on 
the measured deviations, their associated uncertainties and the rules for a verification (JCGM, 2012), the test centre decide whether or not an instrument transformer is within its accuracy class.

In this work, the ratio error and phase displacement of instrument transformers are presented and discussed. Moreover, the standard measuring systems used at PTB for the calibration of the test equipment of test centres and of the measurement uncertainties achieved is illustrated. Finally, an uncertainty budget for the assessment of an instrument transformer in a state-approved test centre or a notified body is calculated as an example (Mohns and Raether, 2017).

\section{Conventional instrument transformers and definition of the deviation of transformers}

Instrument transformers are used to scale a high primary quantity $A_{\mathrm{p}}$ (primary voltage $U_{\mathrm{p}}$ in the case of voltage transformers, primary current $I_{\mathrm{p}}$ in the case of current transformers) as a small secondary quantity $A_{\mathrm{s}}\left(U_{\mathrm{s}}, I_{\mathrm{s}}\right)$ that is easily measurable for meters. The ratio $A_{\mathrm{p}} / A_{\mathrm{s}}$ is the transformer ratio of the instrument transformer. Ideally, the ratio of the primary to the secondary quantity is exactly proportional and in phase. The actual ratio then corresponds to the rated transformer ratio $K_{n}=A_{\mathrm{pn}} / A_{\mathrm{sn}}$ (Ramm et al., 1998). The instrument transformers used in Germany, but also in large parts of Europe, are mainly conventional transformers that work inductively, i.e. according to the principle of a classical transformer. The secondary rated values are usually normalized to $100 \mathrm{~V}$ for voltages and to 1 or $5 \mathrm{~A}$ for currents.

In practice, however, deviations always occur in the magnitude and the phase. These are called ratio error $\varepsilon$ and phase displacement $\delta$. Figure 1a shows the phasor diagram of the actual secondary quantity, $A_{\mathrm{s}}$, of the ideally secondary quantity $A_{\mathrm{p}} / K_{n}$ and of the resulting phase displacement $\delta$ between these quantities. The complex deviation $\Delta A_{\mathrm{s}}$ is also shown. If the phasors are normalized to $A_{\mathrm{p}} / K_{n}$ (Fig. 1b), it then geometrically yields the ratio error $\varepsilon$ as the difference between the vector length and the value 1 . It is as follows:

$$
\begin{gathered}
\varepsilon=\frac{\left|A_{\mathrm{s}}\right|}{\frac{\left|A_{\mathrm{p}}\right|}{K_{n}}}-1=\frac{A_{\mathrm{s}}}{\frac{A_{\mathrm{p}}}{K_{n}}}-1 \\
\delta=\arg \left\{A_{\mathrm{s}}\right\}-\arg \left\{A_{\mathrm{p}}\right\} .
\end{gathered}
$$

The complex measurement error $E$ is also shown with its real part $\alpha$ and its imaginary part $\beta$; it is not really relevant for the definition of these transformer measurement errors. This complex measurement error, however, allows measurement procedures according to the difference method to be derived graphically. It becomes immediately understandable that in the case of small phase displacements (as are required for instrument transformers), the quantities $\varepsilon$ and $\alpha$, as well as $\delta$ and $\beta$, practically agree.

The sources of the measurement errors of instrument transformers are easily derived from the equivalent circuit of a
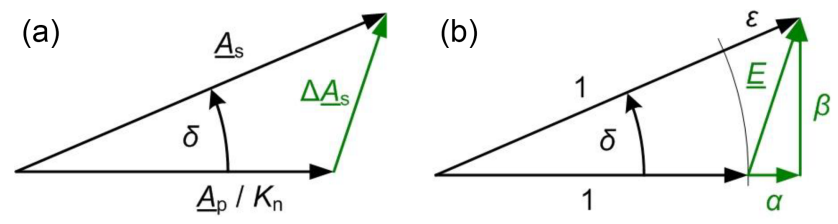

Figure 1. (a) Phasor diagram of an instrument transformer with converted primary quantity $A_{\mathrm{p}} / K_{n}$, secondary quantity $A_{\mathrm{s}}$, and phase displacement $\delta$. The complex difference $\Delta A_{\mathrm{S}}$ is shown in green. (b) Phasor diagram following from the figure on the left by normalization with $A_{\mathrm{p}} / K_{n}$, with the ratio error $\varepsilon$ and the complex measurement error $E$ with the associated real part $\alpha$ and imaginary part $\beta$ (green).

(a)

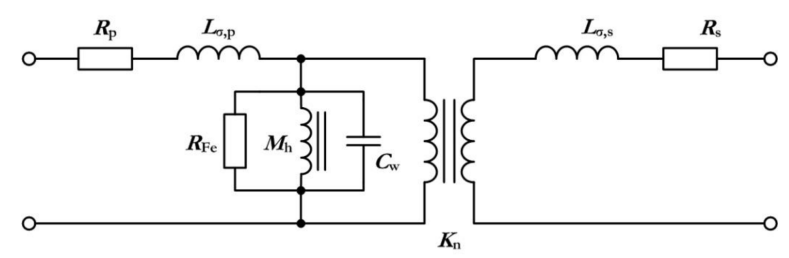

(b)

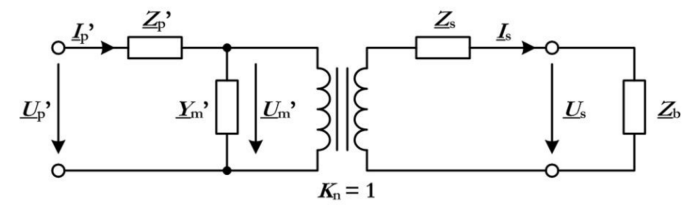

Figure 2. (a) Equivalent circuit of an instrument transformer with discrete components. (b) simplified $T$-equivalent circuit with primary and secondary longitudinal impedance, transversal impedance, and burden $Z_{\mathrm{b}}$.

transformer as shown in Fig. 2a and from the impedances of the $T$-equivalent circuit compiled from this and converted into those of the secondary side as shown in Fig. $2 b$. The values are converted by means of the squared turns ratio $u^{2}=\left(N_{\mathrm{p}} / N_{\mathrm{s}}\right)^{2}$. The corresponding longitudinal and transversal impedances are then given as the following:

$$
\begin{aligned}
Y_{\mathrm{m}}^{\prime} & =\frac{1}{R_{\mathrm{Fe}}^{\prime}}+\mathrm{j} \cdot \omega \cdot\left(C_{\mathrm{w}}^{\prime}-\frac{1}{\omega^{2} \cdot M_{\mathrm{h}}^{\prime}}\right), \\
Z_{\mathrm{p}}^{\prime} & =R_{\mathrm{p}}^{\prime}+j \cdot \omega \cdot L_{\sigma, \mathrm{p}}^{\prime}, \\
Z_{\mathrm{s}} & =R_{\mathrm{s}}+j \cdot \omega \cdot L_{\sigma, \mathrm{s}},
\end{aligned}
$$

where it may be assumed that only the iron loss resistance $R_{\mathrm{Fe}}$ and the main inductance $M_{\mathrm{h}}$ depend on the signal amplitude as a result of the properties of the magnetic core material used. An equivalent winding capacitance $C_{\mathrm{w}}$ is also taken into account; it is relevant mainly for voltage transformers with a high primary number of windings. 
For a voltage transformer, the complex measurement error $E_{0}$ yielded as the no-load error for $Z_{\mathrm{b}} \rightarrow \infty$ is thus

$$
\begin{aligned}
E_{0} & \approx \varepsilon_{\mathrm{u}, 0}+\mathrm{j} \delta_{\mathrm{u}, 0}=\frac{U_{\mathrm{s}}}{U_{\mathrm{p}}^{\prime}}-1=\frac{U_{\mathrm{m}}^{\prime}}{U_{\mathrm{p}}^{\prime}}-1=\frac{1}{1+Y_{\mathrm{m}}^{\prime} Z_{\mathrm{p}}^{\prime}}-1, \\
E_{0} & \approx-Y_{\mathrm{m}}^{\prime} Z_{\mathrm{p}}^{\prime}=-\frac{R_{\mathrm{p}}^{\prime}}{R_{\mathrm{Fe}}^{\prime}}-\frac{L_{\sigma, \mathrm{p}}^{\prime}}{M_{\mathrm{h}}^{\prime}}+\omega^{2} L_{\sigma, \mathrm{p}}^{\prime} C_{\mathrm{w}}^{\prime} \\
& +\mathrm{j} \omega\left(-\frac{L_{\sigma, \mathrm{p}}^{\prime}}{R_{\mathrm{Fe}}^{\prime}}-R_{\mathrm{p}}^{\prime} C_{\mathrm{w}}^{\prime}+\frac{R_{\mathrm{p}}^{\prime}}{\omega^{2} M_{\mathrm{h}}^{\prime}}\right),
\end{aligned}
$$

and in case of an additional load in the form of a burden, the additional error $\Delta E_{\mathrm{b}}$ is obtained by

$$
\begin{aligned}
& \Delta E_{\mathrm{b}}=-\frac{Z_{\mathrm{p}}^{\prime}+Z_{\mathrm{s}}}{Z_{\mathrm{b}}}, \\
& \Delta \varepsilon_{\mathrm{b}}=\operatorname{Re}\left\{\Delta E_{\mathrm{b}}\right\}, \\
& \Delta \delta_{\mathrm{b}}=\operatorname{Im}\left\{\Delta E_{\mathrm{b}}\right\} .
\end{aligned}
$$

The total ratio error and phase displacement is

$\varepsilon_{\mathrm{u}}=\varepsilon_{\mathrm{u}, 0}+\Delta \varepsilon_{\mathrm{b}}$

$\delta_{\mathrm{u}}=\delta_{\mathrm{u}, 0}+\Delta \delta_{\mathrm{b}}$.

From this, it is possible to derive the following principleinduced properties for voltage transformers at power frequency and without turns correction:

- The open-circuit error is negative $\left(-R_{\mathrm{p}}^{\prime} / R_{\mathrm{Fe}}^{\prime}\right)$.

- The phase displacement is usually positive $\left(+R_{\mathrm{p}}^{\prime} / \omega M_{\mathrm{h}}^{\prime}\right)$.

- If, however, capacitive current dominates via $C_{\mathrm{w}}$ compared to inductive current via $M_{\mathrm{h}}$ (high winding number on the primary side), then all relations are inversed.

- The introduction of a burden leads to an even more negative error, which is practically independent of the signal amplitude of the voltage transformer.

For a current transformer, the complex measurement error $E$ results in

$$
\begin{aligned}
E & \approx \varepsilon_{i}+\mathrm{j} \delta_{i}=\frac{I_{\mathrm{s}}}{I_{\mathrm{p}}^{\prime}}-1=\frac{Z_{\mathrm{m}}^{\prime}}{Z_{\mathrm{m}}^{\prime}+Z_{\mathrm{s}}+Z_{\mathrm{b}}}-1 \\
& =\frac{1}{1+Y_{\mathrm{m}}^{\prime}\left(Z_{\mathrm{s}}+Z_{\mathrm{b}}\right)}-1, \\
E & \approx-Y_{\mathrm{m}}^{\prime}\left(Z_{\mathrm{s}}+Z_{\mathrm{b}}\right)=E_{0}+\Delta E_{\mathrm{b}},
\end{aligned}
$$

where

$$
\begin{aligned}
& E_{0} \approx \varepsilon_{\mathrm{i}, 0}+\mathrm{j} \delta_{\mathrm{i}, 0}=-Y_{\mathrm{m}}^{\prime} Z_{\mathrm{s}}=-\frac{R_{\mathrm{s}}}{R_{\mathrm{Fe}}^{\prime}}-\frac{L_{\sigma, \mathrm{s}}}{M_{\mathrm{h}}^{\prime}} \\
&+\omega^{2} L_{\sigma, \mathrm{s}} C_{\mathrm{w}}^{\prime}+\mathrm{j} \omega\left(-R_{\mathrm{s}} C_{\mathrm{w}}^{\prime}-\frac{L_{\sigma, \mathrm{s}}}{R_{\mathrm{Fe}}^{\prime}}+\frac{R_{\mathrm{s}}}{\omega^{2} \cdot M_{\mathrm{h}}^{\prime}}\right), \\
& \Delta E_{\mathrm{b}}=-Y_{\mathrm{m}}^{\prime} Z_{\mathrm{b}} .
\end{aligned}
$$

The terms with $C_{\mathrm{w}}$ are, however, practically negligible. The consequence is that current transformers usually exhibit a wide band behaviour and still transmit frequency fractions in the range of a few kHz with sufficient accuracy. For current transformers, the no-load operation properties at supply frequency are nearly identical with those of voltage transformers. The application of a burden also leads to negative errors; in this case, however, the error is no longer independent of the signal amplitude. Especially at small measuring points ( 1 to $5 \% I_{n}$ ), the permeability of the iron core is considerably smaller. This leads to a reduced inductance $M_{\mathrm{h}}$ which, in turns, results in more significant changes $\Delta E_{\mathrm{b}}$ than at full signal amplitude (20 to $200 \% I_{n}$ ).

\section{Testing equipment and traceability at PTB}

Instrument transformers that are admissible for verification belong to the accuracy classes $0.5-0.2-0.1$ (both current and voltage transformers), or 0.5 or $0,2 \mathrm{~S}$ (current transformers only). Every single instrument transformer that is admissible for verification is tested by a state-approved test centre for measuring instruments for electricity or by a notified body before being mounted into the network in order to find out whether it complies with all the requirements laid down in the type-approval certificate (conformity assessment). The accuracy test is carried out to answer the question as to whether the instrument transformer complies with the maximum permissible error on verification (PTB, 1979).

The tests are carried out by comparison with instrument transformers of "higher quality" - so-called standard transformers. Their ratio error amounts to less than $0.02 \%$, which makes them 10 times as accurate as, for instance, a class $0,2 \mathrm{~S}$ transformer. The comparing instrument in a transformer measuring system is a transformer measuring bridge. The secondary quantities of the instrument transformer to be tested and of the standard transformer are connected to this bridge. From these quantities, the bridge determines the ratio error and the phase displacement of the instrument transformer compared to those of the standard transformer. The instrument transformer is thereby subjected to a test burden which corresponds to the measurement burden and, usually, to one-quarter of the burden of the transformer. For the test equipment, the standard transformer, the transformer measuring bridge, and the test burden, PTB supplies high-precision calibration systems which will be briefly described together with their main properties.

\subsection{Test equipment for standard transformers}

The transformer measuring bridge used at PTB for current and voltage transformers works according to the difference method, which consists of measuring the difference between the secondaries of the PTB's standard and the transformer under test. Figure 3 illustrates this principle with the example of the measurement of current transformers (Kahmann et al., 2017; Ramm and Moser, 1995; Xu, 2015); the same ap- 


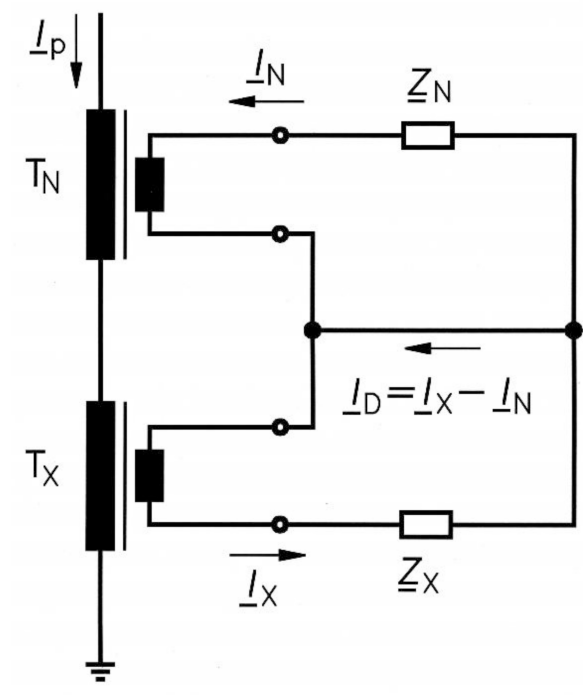

Figure 3. Principle of a measuring bridge for current transformers according to the difference method.

plies analogously to the measurement of voltage transformers (Ramm and Moser, 1996; Badura, 2015).

PTB's standard current transformer $T_{N}$ and current transformer $T_{X}$ under test are connected in series on the primary side and supplied with the desired primary current $I_{\mathrm{p}}$ by the current source. Thereby, the same transformer ratio $K_{n}$ is selected for $T_{N}$ as for $T_{X}$ in order to attain secondary currents $I_{X}$ and $I_{N}$ that are roughly identical. Analogously to Fig. 1, the transformer measuring bridge determines the complex current $I_{N}$ and the differential current $I_{\mathrm{D}}=I_{X}-I_{N}$ by means of suitable, highly sensitive current sensors and a twochannel sampling system. The sampled values are then resolved into spectral values according to their magnitude and phase angle (i.e. complex resolving) in the frequency range with a discrete Fourier transform. From the values at the fundamental frequency, it is then possible to calculate the complex transformer difference $E_{\mathrm{D}}=I_{\mathrm{D}} / I_{N}$ between $T_{X}$ and $T_{N}$, and from this difference the ratio error $\varepsilon_{\mathrm{D}}$ and the phase displacement $\delta_{\mathrm{D}}$ can be calculated. Here, it is important to keep in my mind that these errors still contain the low errors of PTB's standard transformer. The errors of $T_{X}$ with the correction for $T_{N}$ are:

$\varepsilon_{X}=\varepsilon_{\mathrm{D}}+\varepsilon_{N}$,

$\delta_{X}=\delta_{\mathrm{D}}+\delta_{N}$.

The current transformer under test is thereby subjected to a burden $Z_{X}$ in such a way that this burden corresponds to the load due to the measurement leads and the transformer measuring bridge at the test centre. The standards used by PTB for calibration are current comparators whose measurement errors are in the range between less than $10^{-6}$ and $10^{-5}$ (Kusters and Moore, 1964; Mohns et al., 2017). The range of the primary rated currents of these references is between 0.1

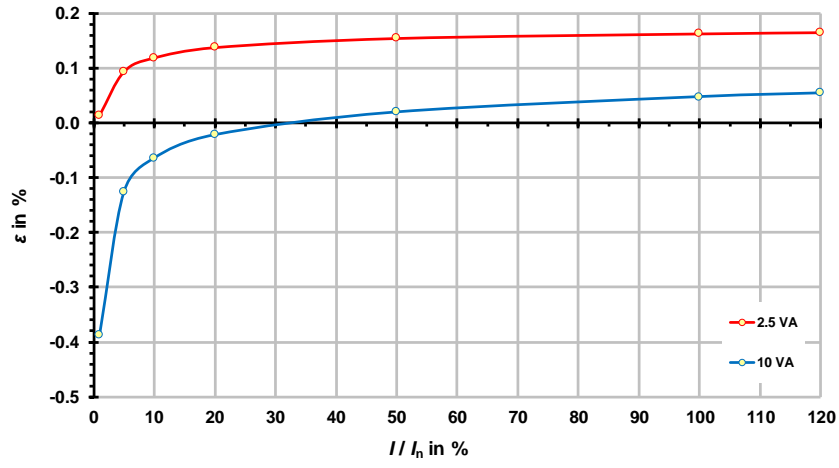

Figure 4. Ratio error of a class $0,2 \mathrm{~S}$ current transformer (Mohns, 2018).

and $60000 \mathrm{~A}$. The measurement uncertainty that is typically assigned when a standard current transformer is calibrated for the verification of instrument transformers is approximately $\pm 0.003 \%$ or 0.003 crad (centi-radian, which corresponds to $0.1^{\prime}$, angular minute), with a confidence interval of approx. $95 \%$ corresponding to $k=2$.

The relations stated for PTB's calibration facility for current transformers can, in principle, be applied analogously to the calibration facility for voltage transformers. It is also based on the difference method. Two voltage transformers connected in parallel on the primary side are connected to the primary voltage $U_{\mathrm{p}}$, and, on the secondary side, to the voltage transformer measuring bridge. The secondary voltage $U_{N}$ of the transformer used as a reference $T_{N}$, and the difference voltage $U_{\mathrm{D}}=U_{X}-U_{N}$ are detected by means of suitable voltage sensors (complex detection). Similar to the current transformer measuring system, the transformer measurement of $T_{X}$ is calculated according to Eq. (8) from the complex measurement error $E_{\mathrm{D}}=U_{\mathrm{D}} / U_{N}$. A set of standard voltage transformers at PTB provide a range of the primary rated voltages from $100 \mathrm{~V}$ up to $400 / \sqrt{ } 3 \mathrm{kV}$. The measurement uncertainties assigned for calibrating standard voltage transformers are also $0.003 \%$ or $0.003 \mathrm{crad}(k=2)$.

\subsection{Test equipment for standard burdens}

During the accuracy test, standard burdens simulate the load that will later be induced in the network by the instrument transformer due to the energy meters and cabling connected to the secondary side. Characteristics of a burden are the rated apparent power $S_{n}$ and the rated power factor $\cos \beta_{n}$. The deviation between the actual apparent power and $S_{n}$ may not exceed $3 \%$; for the phase angle $\beta$, the limit amounts to \pm 3 crad on the basis of $\beta_{n}$ (PTB, 1979).

The standard values of the burden steps for the verification of current transformers are based on the rated power values for transformers that are admissible for verification and are usually in the range between 1 and $30 \mathrm{VA}$. The burden power factor $\cos \beta$ describes the ratio of active to the ap- 
Table 1. Description of the symbols used.

\begin{tabular}{lll}
\hline Quantity & Description & Unit \\
\hline$\varepsilon_{X}$ & Result: ratio error of the transformer $(X)$ & $\%$ \\
$\varepsilon_{N}$ & Ratio error of the standard $(N)$ & $\%$ \\
$\varepsilon_{\mathrm{D}}$ & Indicated difference of the bridge for the ratio error $X-N$ & $\%$ \\
$d_{\mathrm{WM}}$ & Uncertainty in the transformer measuring bridge & $\%$ \\
$d_{\mathrm{MP}}$ & Influence of the test point & $\%$ \\
$s_{\mathrm{B}}$ & Sensitivity of the transformer $(X)$ due to a burden change & $\% \mathrm{VA}^{-1}$ \\
$\Delta_{\mathrm{B}}$ & Error of the burden: measured burden - rated burden & $\mathrm{VA}^{-1}$ \\
\hline
\end{tabular}

Table 2. Uncertainty budget of the ratio error for a class $0,2 \mathrm{~S}$ instrument transformer at a signal amplitude $I_{n}$ of $100 \%$ and a rated burden $S_{n}=10 \mathrm{VA}$.

\begin{tabular}{|c|c|c|c|c|c|c|}
\hline Quantity & Value & Standard uncertainty $u$ & Distribution & Sensitivity coefficient $c$ & Variance $(c u)^{2}$ & Index in $\%$ \\
\hline$\varepsilon_{N}$ & $0.000 \%$ & $0.0015 \%$ & normal & 1 & $2.25 x^{-6}$ & 1.6 \\
\hline$\varepsilon_{\mathrm{D}}$ & $0.048 \%$ & $0.0003 \%$ & normal & 1 & $9.00 x^{-8}$ & 0.1 \\
\hline$d_{\mathrm{WM}}$ & $0 \%$ & $0.0115 \%$ & rectangular & 1 & $1.32 x^{-4}$ & 93.5 \\
\hline$d_{\mathrm{MP}}$ & $0 \%$ & $0.0003 \%$ & rectangular & 1 & $9.00 x^{-8}$ & 0.1 \\
\hline$s_{\mathrm{B}}$ & $-0.015 \% \mathrm{VA}^{-1}$ & & - & & & \\
\hline \multirow[t]{2}{*}{$\Delta_{\mathrm{B}}$} & $0 \mathrm{VA}$ & $0.1730 \mathrm{VA}$ & rectangular & $-0.015 \% \mathrm{VA}^{-1}$ & $6.73 x^{-6}$ & 4.8 \\
\hline & & & & Sum & $1.41 x^{-4}$ & \\
\hline$\varepsilon_{x}$ & $0.048 \%$ & & & & $U=0.024$ & $\%(k=2)$ \\
\hline
\end{tabular}

parent power. Below $5 \mathrm{VA}$, the load is purely ohmic; at $5 \mathrm{VA}$ and above, the load is ohmic-inductive, i.e. the voltage phasor precedes the current phasor (by $36.9^{\circ}$ at $\cos \beta=0.8$ ). The standard values of the burden steps for the verification of voltage transformers cover a vast range that extends to up to $300 \mathrm{VA}$.

PTB's measuring facility for standard burdens (Braun et al., 1993; Ni, 2015) is based on the measurement of the complex impedance via the ratio of the voltage $U$ to the current $I$. Suitable sensors are connected in such a way that no loading of the burden is caused by the sensors. They cover the range from $1 \mathrm{~mA}$ to $10 \mathrm{~A}$ (current sensors) and from a few millivolts to $300 \mathrm{~V}$ (voltage sensors). An analogue power amplifier supplies a test power of up to $700 \mathrm{VA}$. The achievable measurement uncertainty with this calibrator is from 0.02 to $0.05 \%$. The measurement uncertainties attributed to standard burdens are, however, higher - from 0.1 to $0.5 \%(k=2)$. This is due to the properties of the burden under test determined during the calibration, such as the stability (influences of heating up) or the repeatability.

\subsection{Test equipment for transformer measuring bridges}

The transformer measuring bridge for the verification (conformity assessment) of an instrument transformer compares either the secondary currents $I_{X}$ and $I_{N}$ or the secondary voltages $U_{X}$ and $U_{N}$ of the transformer under test and of the standard transformer according to the magnitude and the phase, pursuant to the definitions Eq. (1). The differences between $X$ and $N$ which should hereby be shown by the measuring bridge are in the range of up to $\pm 1.5 \%$ and $\pm 2.7 \mathrm{crad}$ (class $0.5 \mathrm{~S}$ at $1 \% I_{n}$ ). Hereby, the admissible deviations according to PTB (1979) are based on the maximum permissible error limits of a class $0,2 \mathrm{~S}$ current transformer. They amount to one-tenth of the error limits required there, i.e. $\pm 0.02 \%$ ( $\pm 0.03 \mathrm{crad})$ in the case of measuring bridges for voltage transformers, and at test points of 20 to $200 \%$ in the case of measuring bridges for current transformers. In addition, increased limits of $\pm 0.035 \%( \pm 0.045 \mathrm{crad})$ and $\pm 0.075 \%$ ( $\pm 0.09 \mathrm{crad})$, respectively, are permitted for the test points 5 and $1 \%$.

The calibrator for transformer measuring bridges (Ramm et al., 1998) works according to the principle of feeding errors divided into a real and an imaginary part (see Fig. 1) by means of high-precision, electronically error-compensated current transformers or voltage dividers. The measurement uncertainties achieved with this calibration system are another 10 times lower than the tolerance values required for transformer measuring bridges under test. The working range covers currents of up to $10 \mathrm{~A}$ for $I_{X}$ and $I_{N}$, and secondary voltages of more than $200 \mathrm{~V}$ for $U_{X}$ and $U_{N}$. 
4 Uncertainty budget for the calibration of an instrument transformer

\subsection{Simplified methodology of measurement uncertainty calculation}

The basis of the measurement uncertainty analysis consists of statistically obtained findings on error propagation. Contrary to a worst case estimation, in which the error propagation of the maximum error is calculated somewhat conservatively, measurement error calculation is based on the Gaussian error propagation. The basic approach consists of identifying the physical model of a system and transforming it into a mathematically utilizable calculation model in the form of an analytical equation. Very generally, the model equation $y$ obtained is a function of several variables $x_{1}, x_{2}, x_{3} \ldots$ with $y=f\left(x_{1}, x_{2}, x_{3}, \ldots\right)$.

The uncertainty $u\left(x_{i}\right)$ of a variable $x_{i}$ obviously influences the result $y$. This result exhibits a deviation by the difference $\partial y\left(u\left(x_{i}\right)\right)$. This deviation, or rather this indeterminacy $\partial y\left(u\left(x_{i}\right)\right)$, is

$\partial y\left(u\left(x_{i}\right)\right)=\frac{\partial f\left(x_{1}, x_{2}, x_{3}, \ldots\right)}{\partial x_{i}} \cdot u\left(x_{i}\right)$,

and derives from linearization around a working point by means of a Taylor series which is interrupted after the first order. The sensitivity coefficient $c_{i}$ corresponds to the differential quotient $\partial f / \partial x_{i}$. Its sole purpose is improved legibility. The total uncertainty $u(y)$ is thus the geometrical addition ${ }^{1}$ of all individual uncertainties $\partial y\left(u\left(x_{i}\right)\right)$ :

$$
\begin{aligned}
u_{\mathrm{B}}(y) & =\sqrt{\sum\left(\partial y\left(u\left(x_{i}\right)\right)\right)^{2}} \\
& =\sqrt{\left(c_{1} \cdot u\left(x_{1}\right)\right)^{2}+\left(c_{2} \cdot u\left(x_{2}\right)\right)^{2}+\left(c_{3} \cdot u\left(x_{3}\right)\right)^{2}+\ldots} .
\end{aligned}
$$

This measurement uncertainty is a so-called type B uncertainty. In addition, the type A contribution to the total uncertainty will later be added geometrically to the type B uncertainty. Type A uncertainty essentially describes the statistical variability in the mean value $y_{\text {ave }}$ of the measurand $y$ to be determined which is yielded over the course of the measurement. The estimated quantity for type A measurement uncertainty is obtained by means of the standard deviation $s(y)$, the number $N$ of measured values, and Student's $t$ factor $t$ (DOF, $P)$ as

$$
\begin{aligned}
& u_{\mathrm{A}}\left(y_{\mathrm{ave}}\right)=t(\mathrm{DOF}, P) \cdot \frac{s(y)}{\sqrt{N}}=\frac{t(\mathrm{DOF}, P)}{\sqrt{N}} . \\
& \sqrt{\frac{1}{N-1} \cdot \sum_{i=1}^{N}\left(y_{i}-y_{\mathrm{ave}}\right)^{2} .}
\end{aligned}
$$

Student's $t$ factor is listed in the table for the different confidence intervals $P$ and the degrees of freedom $\mathrm{DOF}=N-1$

\footnotetext{
${ }^{1}$ This is applicable in cases where no correlation between variables $x_{i}$ exist.
}

Table 3. Description of the symbols used.

\begin{tabular}{lll}
\hline Quantity & Description & Unit \\
\hline$\delta_{X}$ & $\begin{array}{l}\text { Result: phase displacement of the } \\
\text { transformer }(X)\end{array}$ \\
& $\begin{array}{l}\text { phase displacement of the standard }(N) \\
\delta_{N}\end{array}$ &, \\
$\delta_{\mathrm{D}}$ & $\begin{array}{l}\text { Indicated difference of the bridge } \\
\text { for the phase } \\
\text { displacement } X-N\end{array}$ &, \\
& Uncertainty in the transformer \\
$d_{\mathrm{WM}}$ & $\begin{array}{l}\text { measuring bridge } \\
\text { Influence of the test point }\end{array}$ \\
$d_{\mathrm{MP}}$ & $\begin{array}{l}\text { Sensitivity of the transformer }(X) \\
\text { due to burden change }\end{array}$ \\
$s_{\mathrm{B}}$ & $\begin{array}{l}\text { Error of the burden: measured burden }- \\
\Delta_{\mathrm{B}}\end{array}$ & $\mathrm{VA}^{-1}$ \\
& rated burden
\end{tabular}

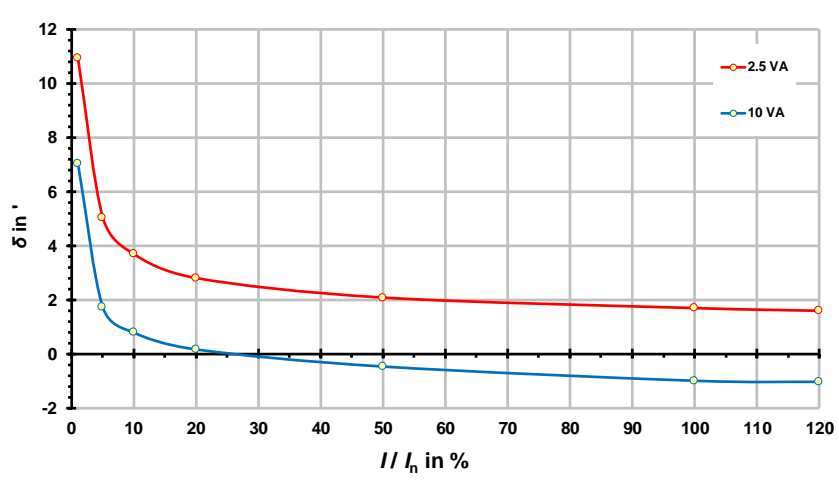

Figure 5. Phase displacement of a class $0,2 \mathrm{~S}$ current transformer (Mohns, 2018).

(GUM, 2008). For a sufficient number of measured values $(N>10)$, this factor is, however, approx. 2 for a confidence interval of $95 \%$, and approx. 1 for the confidence interval of approx. $68 \%$ which is relevant for calculating the standard uncertainty.

\subsection{Example of an uncertainty budget for class $0,2 \mathrm{~S}$ current transformer}

\subsubsection{Uncertainty budget for the ratio error}

First, the model equation for the ratio error $\varepsilon_{X}$ must be developed. According to Eq. (8), $\varepsilon_{X}=\varepsilon_{\mathrm{D}}+\varepsilon_{N}$. Influences due to the unprecise setting of the test point (e.g. $99 \%$ instead of $100 \%$ ) and of the tolerances of the standard burden used have to be taken into account. The model equation then expands into

$\varepsilon_{X}=\varepsilon_{\mathrm{D}}+\varepsilon_{N}+d_{\mathrm{WM}}+d_{\mathrm{MP}}+s_{\mathrm{B}} \cdot \Delta_{\mathrm{B}}$.

The example stated refers to the $100 \%$ testing point of a class $0,2 \mathrm{~S}$ current transformer. For the standards, the uncertainty contributions are used as those that are determined 
Table 4. Uncertainty budget of the phase displacement for a class $0,2 \mathrm{~S}$ instrument transformer at $100 \% I_{n}$ and $S_{n}=10 \mathrm{VA}$.

\begin{tabular}{|c|c|c|c|c|c|c|}
\hline Quantity & Value & Standard uncertainty $u$ & Distribution & Sensitivity coefficient $c$ & Variance $(c u)^{2}$ & Index in $\%$ \\
\hline$\delta_{N}$ & $0.00^{\prime}$ & $0.05^{\prime}$ & normal & 1 & $2.50 x^{-3}$ & 0.7 \\
\hline$\delta_{\mathrm{D}}$ & $-1.00^{\prime}$ & $0.05^{\prime}$ & normal & 1 & $2.50 x^{-3}$ & 0.7 \\
\hline$d_{\mathrm{WM}}$ & $0^{\prime}$ & $0.58^{\prime}$ & rectangular & 1 & $3.33 x^{-1}$ & 97.3 \\
\hline$d_{\mathrm{MP}}$ & $0^{\prime}$ & $0.02^{\prime}$ & rectangular & 1 & $2.99 x^{-4}$ & 0.1 \\
\hline$s_{\mathrm{B}}$ & $-0.36^{\prime} \mathrm{VA}^{-1}$ & & - & & & \\
\hline \multirow[t]{2}{*}{$\Delta_{\mathrm{B}}$} & $0 \mathrm{VA}$ & $0.17 \mathrm{VA}$ & rectangular & $-0.360^{\prime} \mathrm{VA}^{-1}$ & $3.88 x^{-3}$ & 1.1 \\
\hline & & & & Sum & $3.42 \times^{-1}$ & \\
\hline$\delta_{x}$ & $-1.0^{\prime}$ & & & & $U=1.2$ & ${ }^{\prime}(k=2)$ \\
\hline
\end{tabular}

from the measured values and associated uncertainties (for standard transformers) that are documented in PTB's test certificates, from the admissible limit values (transformer measuring bridge, standard burden), and from the results obtained during the testing of the instrument transformer (see Fig. 4). The thus obtained results of this uncertainty budget can also be used at signal amplitudes of $>20 \% I_{n}$. Only for the testing points 1 and $5 \%$ is it recommended that this uncertainty budget be recalculated using the modified numerical values. Table 1 lists and explains the quantity symbols used in Eq. (9).

The following conditions and numerical values are given:

- The transformer $(X)$ is loaded with the rated burden $10 \mathrm{VA}$.

- The mean value of the display of the transformer measuring bridge $\varepsilon_{\mathrm{D}}=0.048 \%$ from $N=10$ measured values. The standard deviation $s$ of the displayed value is $\pm 0.001 \%$, from which the type A standard uncertainty $u=s / \sqrt{ } N= \pm 0.0003 \%$ is calculated.

- Measurement error of the standard $\varepsilon_{N}=0.000 \%$. The expanded measurement uncertainty in the standard is stated as being $U= \pm 0.003 \%(k=2)$. The standard uncertainty is $u=U / 2= \pm 0.0015 \%$.

- Via the admissible tolerance of $a= \pm 0.02 \%$, the uncertainty in the transformer measuring bridge is considered as having a rectangular distribution. The standard uncertainty is $u=a / \sqrt{ } 3= \pm 0.0115 \%$.

- The influence of the measuring point is calculated via one measurement at 99.0 and one at $100.0 \%$. From the measured ratio error difference of $0.005 \%$, a maximum change of $10 \%$, i.e. $a= \pm 0.0005 \%$ with a rectangular distribution, is estimated for the instrument transformer, taking a tolerance of the testing point indication of $\pm 0.1 \%$ into account. The standard uncertainty is $u=a / \sqrt{ } 3= \pm 0.0003 \%$.

- The burden sensitivity of the transformer is determined via the measurement at one-quarter of the burden, which is necessary anyway. Due to the measurement with 10 and $2.5 \mathrm{VA}$, the value indicated by the measuring facility changes from $\varepsilon_{\mathrm{D}}=0.048 \%$ (10 VA) to $\varepsilon_{\mathrm{D}}=+0.163 \% \quad(2.5 \mathrm{VA})$. The sensitivity is thus $s_{\mathrm{B}}=(0.048-0.163 \%) /(10$ $2.5 \mathrm{VA})=-0.015 \% \mathrm{VA}^{-1}$. The admissible tolerance of the standard burden is $a= \pm 3 \%$ (with a rectangular distribution), corresponding to $\pm 0.3 \mathrm{VA}$. The standard uncertainty in the burden is $u=a / \sqrt{ } 3= \pm 0.173 \mathrm{VA}$.

The uncertainty budget presented in Table 2 is derived from these assumptions and numerical values. This table makes several results obvious. Firstly, the result for the instrument transformer is indicated as $\varepsilon_{X}=0.048 \%$. The expanded measurement uncertainty amounts to $U\left(\varepsilon_{X}\right)= \pm 0.024 \%$ for $k=2$, i.e. for a confidence interval of $95 \%$. Secondly, it appears that the greatest influence on the measurement uncertainty is caused by the measuring bridge (index $93.5 \%$ ). By using the actual measurement errors determined and the measurement uncertainties instead of the permissible tolerances of $\pm 0.02 \%$ stated in the calibration certificate of the measuring bridge, it might be possible to reduce the uncertainty contribution $u\left(d_{\text {Bridge }}\right)$ to 0.003 to $0.005 \%$. It must, however, be checked for each individual case whether the individual measuring bridge really allows these improved measurement uncertainties at all. In any case, at smaller test points (1\%) and with class $0.5 \mathrm{~S}$ transformers, for instance, these relations change. It can be assumed that the greatest influence is caused from the greater burden sensitivity of the transformer.

\subsubsection{Uncertainty budget for the phase displacement}

Similar to the procedure described in Sect. 4.2.1 for the uncertainty in the ratio error, the model equation for the phase displacement $\delta_{X}$ must be developed. According to Eq. (8), $\delta_{X}=\delta_{\mathrm{D}}+\delta_{N}$. Influences due to the unprecise setting of the test point and of the tolerances of the standard burden used have to be taken into account. The model equation then expands to

$\delta_{X}=\delta_{\mathrm{D}}+\delta_{N}+d_{\mathrm{WM}}+d_{\mathrm{MP}}+s_{\mathrm{B}} \cdot \Delta_{\mathrm{B}}$. 
The example stated refers to the $100 \%$ testing point of a class $0,2 \mathrm{~S}$ current transformer. For the standards, the uncertainty contributions are used as those that are determined from the measured values and associated uncertainties (for standard transformers) that are documented in PTB's test certificates, from the admissible limit values (transformer measuring bridge, standard burden), and from the results obtained during the testing of the instrument transformer (see Fig. 5). The thus obtained results of this uncertainty budget can also be used at signal amplitudes of $>20 \% I_{n}$. Only for the testing points 1 and $5 \%$ is it recommended that this uncertainty budget be recalculated using the modified numerical values. Table 3 lists and explains the quantity symbols used in Eq. (10).

The following conditions and numerical values are given:

- The transformer $(X)$ is loaded with the rated burden $10 \mathrm{VA}$.

- The mean value of the display of the transformer measuring bridge $\delta_{\mathrm{D}}=-1^{\prime}$ from $N=10$ measured values. The standard deviation $s$ of the displayed value is $\pm 0.158^{\prime}$, from which the type A standard uncertainty $u=s / \sqrt{ } N= \pm 0.05^{\prime}$ is calculated.

- Measurement error of the standard $\delta_{N}=0.00^{\prime}$. The expanded uncertainty in the standard is stated as $U= \pm 0.1^{\prime}(k=2)$. The standard uncertainty is $u=$ $U / 2= \pm 0.05^{\prime}$.

- Via the admissible tolerance of $a= \pm 1^{\prime}$, the uncertainty in the transformer measuring bridge is considered as having a rectangular distribution. The standard uncertainty is $u=a / \sqrt{ } 3= \pm 0.5773^{\prime}$.

- The influence of the measuring point is calculated via one measurement at $99.0 \%$ and one at $100.0 \%$. From the measured difference of $0.3^{\prime}$, a maximum change of $10 \%$, i.e. $a= \pm 0.03^{\prime}$ with a rectangular distribution, is estimated for the instrument transformer, taking a tolerance of the testing point indication of $\pm 0.1 \%$ into account. The standard uncertainty is $u=a / \sqrt{ } 3=$ $\pm 0.0173^{\prime}$.

- The burden sensitivity of the transformer is determined via the measurement at one-quarter of the burden, which is necessary anyway. Due to the measurement with $10 \mathrm{VA}$ and $2.5 \mathrm{VA}$, the value indicated by the measuring facility changes from $\delta_{\mathrm{D}}=-1^{\prime}(10 \mathrm{VA})$ to $\delta_{\mathrm{D}}=+1.7^{\prime}$ (2.5 VA). The sensitivity is thus $s_{\mathrm{B}}=\left(-1^{\prime}-1.7^{\prime}\right) /(10$ $2.5 \mathrm{VA})=-0.36^{\prime} \mathrm{VA}^{-1}$. The admissible tolerance of the standard burden is $a= \pm 3 \%$ (with a rectangular distribution), corresponding to $\pm 0.3 \mathrm{VA}$. The standard uncertainty in the burden is $u=a / \sqrt{ } 3= \pm 0.173 \mathrm{VA}$.

The uncertainty budget presented in Table 4 is derived from these assumptions and numerical values. This table makes several results obvious. Firstly, the result for the instrument transformer is indicated as $\delta_{X}=-1^{\prime}$. The expanded measurement uncertainty amounts to $U\left(\varepsilon_{X}\right)= \pm 1.2^{\prime}$ for $k=2$, i.e. for a confidence interval of $95 \%$. Secondly, it appears that the greatest influence on the measurement uncertainty is caused by the measuring bridge (index $97.3 \%$ ). By using the actual measurement errors determined and the measurement uncertainties stated in the calibration certificate of the measuring bridge instead of the permissible tolerances of $\pm 1^{\prime}$, it might be possible to reduce the uncertainty contribution $u\left(\delta_{\text {Bridge }}\right)$ from 0.2 to $0.4^{\prime}$. It must, however, be checked for each individual case whether the individual measuring bridge really allows these improved measurement uncertainties at all. In any case, at smaller test points $(1 \%)$ and with class $0,5 \mathrm{~S}$ transformers, for instance, these relations change. It can be assumed that the greatest influence is caused from the greater burden sensitivity of the transformer.

\subsection{Summary and findings of the presented uncertainty budgets}

The approach introduced here was intended to develop an uncertainty budget which is as simple as possible to elaborate by using permissible tolerances of the test equipment according to the test certificates or according to PTB (1979). The measurement errors (standard transformer) are only corrected where this is necessary or feasible by simple means. In addition, using the permissible tolerances of the bridge and of the standard burden according to PTB (1979) instead of a tighter range, based on the actual measurement errors, provides a margin in the conservatively calculated measurement uncertainty which has thus been overestimated. What is also interesting is the measurement capability index of the accuracy class of the transformer and the measurement uncertainty which is $\approx 8$ (amount $0.2 \% / 0.024 \%$ and phase $10^{\prime} / 1.2^{\prime}$, respectively). This means that the measuring system is approx. 8 times more accurate than the accuracy class of the device under test. There is a consensus (Sommer et al., 2001; JCGM, 2012) that the minimum accepted measurement capability index is 3 . Vice versa, for the verification which determines whether the instrument transformer is within its accuracy class or not, it means that measured ratio error must be within $\pm 7 / 8$ of the class limits to have a margin of $1 / 8$ due to the system's measurement uncertainty. This leads to a widened acceptance interval which greatly lowers the probability of a false rejection of a conformal instrument transformer.

Data availability. The data relevant to this work are the data for the two figures (Figs. 4 and 5; Mohns, 2018).

Competing interests. The authors declare that they have no conflict of interest. 
Special issue statement. This article is part of the special issue "Evaluating measurement data and uncertainty". It is not associated with a conference.

Edited by: Klaus-Dieter Sommer

Reviewed by: two anonymous referees

\section{References}

Badura, H.: Entwicklung einer Spannungswandlermesseinrichtung ESM III, Bachelor paper, Ostfalia Hochschule für angewandte Wissenschaften, Wolfenbüttel, 2015.

Braun, A., Moser, H., and Ramm, G.: Ein neuer Meßplatz zur Prüfung von Meßwandlerbürden, PTB-Mitteilungen, 103, 5/93, 1993.

GUM: JCGM 100:2008: Evaluation of measurement data - Guide to the expression of uncertainty in measurement, available at: www.BIPM.org (last access: 27 April 2018), 2008.

JCGM: JCGM 106:2012: Evaluation of measurement data - The role of measurement uncertainty in conformity assessment, available at: wwww.BIPM.org (last access: 27 April 2018), 2012.

Kahmann, M., Zayer, P., and Arzberger, M.: Handbuch Elektrizitätsmesstechnik, VDE Verlag, 3. Auflage 2017, ISBN: 978-38007-4403-9, 2017.

Kusters, N. L. and Moore, W. J. M.: The compensated current comparator, a new reference standard for current-transformer calibrations in industry, IEEE T. Instrum. Meas., 13, 107-114, 1964.

MEG: Mess- und Eichgesetz 2015 (Measures and Verification Act), available at: www.ptb.de (last access: 27 April 2018), 2015.

Mohns, E.: Dataset: error characteristics of a class 0.2S current transformer for publication (Version v01) [Data set], Zenodo, https://doi.org/10.5281/zenodo.1226877, 2018.
Mohns, E. and Raether, P.: Prüftechnik und deren Einfluss bei der Kalibrierung von Messwandlern, tm - Technisches Messen, 2196-7113, 0171-8096, https://doi.org/10.1515/teme2016-0045, January, 2017.

Mohns, E., Roeissle, G., Fricke, S., and Pauling, F.: An AC Current Transformer Standard Measuring System for Power Frequencies, IEEE T. Instrum. Meas., 66, 1433-1440, https://doi.org/10.1109/TIM.2017.2648918, 2017.

Ni, S.: Entwicklung einer Bürdenmesseinrichtung BMEII, Bachelor paper, Ostfalia Hochschule für angewandte Wissenschaften, Wolfenbüttel, 2015.

PTB: Physikalisch-Technische Bundesanstalt: PTB-Prüfregeln Messwandler, 12, Braunschweig, 1977, with modifications 5/79, 1979.

Ramm, G. and Moser, H.: Eine neuartige, rechnergesteuerte und selbstkalibrierende Stromwandler-Meßeinrichtung, PTBMitteilungen, 105, 4/95, 1995.

Ramm, G. and Moser, H.: Eine neuartige, rechnergesteuerte und selbstkalibrierende Spannungswandler-Meßeinrichtung, PTBMitteilungen, 106, 4/96, 1996.

Ramm, G., Roeissle, G., and Latzel, H.-G.: Rechnergesteuerte Kalibrierung von Meßeinrichtungen für Strom- und Spannungswandler, PTB-Mitteilungen, 108, 3/98, 1998.

Sommer, K. D., Chappell, S. E., and Kochsieck, M.: Calibration and verification: Two procedures having comparable objectives and results, OIML Bulletin, XLII, Number 1, January, 2001.

$\mathrm{Xu}, \mathrm{Q}$.: Entwicklung einer Stromwandlermesseinrichtung SEKAM III, Bachelor paper, Ostfalia Hochschule für angewandte Wissenschaften, Wolfenbüttel, 2015. 